\title{
DER PROZESS DER RELECTURE IN DER JOHANNEISCHEN LITERATUR ${ }^{1}$
}

\author{
JEAN ZUMSTEIN \\ (Dunantstr. 1, CH-8044 Zürich, Switzerland)
}

Die gegenwärtige Exegese nimmt mehrheitlich an, dass die johanneische Literatur in ihrer kanonischen Form (Joh, 1 Joh, 2 Joh, $3 \mathrm{Joh}$ ) das Resultat eines langen Prozesses der Ausgestaltung darstellt, der sich im wesentlichen der Tätigkeit einer Schule verdankt. ${ }^{2}$ Diese Annahme wirft zwei Fragen auf: Wie hat die johanneische Schule die Traditionen, deren Verwalterin sie war, aufgenommen und weitergegeben? Und welcher hermeneutischer Verfahren hat sie sich bedient, um die verschiedenen Ausformungen dieser Traditionen $\mathrm{zu}$ interpretieren und $\mathrm{zu}$ aktualisieren? Im Sinne einer Arbeitshypothese schlagen wir vor, im Prozess der Relecture ${ }^{3}$ eines der wichtigsten Verfahren zu sehen, das die theologische Arbeit der johanneischen Schule auszeichnet. Dieser Prozess der Relecture ist keine Erfindung der johanneischen Kreise; er ist bereits sowohl im Alten Testament, insbesondere in den prophetischen Büchern, ${ }^{4}$ als auch in den paulinischen und synoptischen Traditionen belegt. Das Ziel der folgenden Ausführungen besteht darin, die unterschiedlichen Aspekte dieser Arbeit der Relecture, die wir in der johanneischen Literatur und insbesondere im vierten Evangelium wahrnehmen, zu beschreiben.

\footnotetext{
1 Dieser Vortrag wurde auf französisch als Main Paper anlässlich der 50. SNTS-Generalversammlung am 3. August 1995 in Prag gehalten. Die deutsche Fassung ist dank der unentbehrlichen Hilfe meines Assistenten Andreas Dettwiler zustande gekommen.

2 Zu diesem Punkt siehe den jüngsten Beitrag dazu: U. Schnelle, 'Die johanneische Schule', in: Bilanz und Perspektiven gegenwärtiger Auslegung des Neuen Testaments. Symposion zum 65. Geburtstag von Georg Strecker (hg. von F. W. Horn; BZNW 75; Berlin/New York, 1995) 198-217.

${ }^{3}$ Zum Begriff der Relecture vgl. G. Genette, Palimpsestes. La littérature au second degré (collection Poétique; Paris, 1982): allgemeine Definition siehe insb. 7-14. Es ist jedoch anzumerken, dass Genette nicht mit dem Begriff der Relecture, sondern mit demjenigen der Transtextualität arbeitet.

4 Vgl. O. H. Steck, 'Prophetische Prophetenauslegung', in: Wahrheit der Schrift - Wahrheit der Auslegung. Eine Zürcher Vorlesungsreihe zu Gerhard Ebelings 80. Geburtstag am 6. Juli 1992 (hg. von H. G. Geisser u.a.; Zürich, 1993) 198-244.
} 


\section{DIE ÜBERSCHRIFTEN - HINWEISE AUF EINE ABSCHLIESSENDE RELECTURE}

Zunächst soll eine Definition vorgeschlagen werden. Der Prozess der Relecture liegt dann vor, wenn ein erster Text die Bildung eines zweiten Textes hervorruft und wenn dieser zweite Text seine volle Verständlichkeit erst im Bezug zum ersten Text gewinnt. In diesem Sinn ist das erste Relecture-Phänomen, auf das man bei der Lektüre eines Werkes stösst, seine Überschrift. Die Überschrift eines Werkes - ob vom Autor oder von einem vom Autor verschiedenen Herausgeber - drückt stets eine umfassende Stellungnahme zu diesem Werk aus und lässt beim Lesen auf eine erste Interpretation schliessen. Wie ein Literaturwissenschaftler zutreffend festhält: ' Vor dem Text gibt es den Titel, nach dem Text bleibt der Titel.'5

Die johanneische Forschung hat nie ein besonderes Interesse an den Überschriften der johanneischen Schriften bekundet; ausgenommen davon sind Adolph von Harnack und Theodor Zahn zu Beginn unseres Jahrhunderts und Martin Hengel in jüngerer Zeit. ${ }^{6}$ Dieses Desinteresse lässt sich angesichts der Tatsache, dass bereits sehr alte Manuskripte des Johannesevangeliums - die Papyri

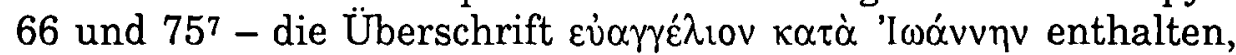
kaum rechtfertigen. Es gibt also gute Gründe für die Annahme, dass die dem vierten Evangelium zugeordnete Überschrift nicht ein später Akt der Herausgabe war, sondern dass sie in die Zeit fällt, als dieses Werk zur Verbreitung freigegeben wurde - d.h. wahrscheinlich in die erste Hälfte des zweiten Jahrhunderts. Es kann leider nicht mehr festgestellt werden, wer diese verlegerische Tat ausgeführt hat. Handelt es sich um die johanneische Schule? Oder handelt es sich um eine Person ausserhalb der johanneischen Kreise? Niemand wird dies mit Gewissheit sagen können. Wie auch immer: Die dem Evangelium, aber auch den drei Briefen

${ }^{5} \mathrm{~F}$. Hallyn/G. Jacques, 'Aspects du paratexte', in: Methodes du texte: introduction aux études littéraires (dir. par M. Delcroix/F. Hallyn; 4e tir.; Paris/Louvain-la-Neuve, 1987) 210 (Zitat von M. Hausser).

${ }^{6}$ Siehe dazu die beiden Studien von M. Hengel, Die Evangelienüberschriften (SHAW.PH; Heidelberg, 1984); ders., Die johanneische Frage. Ein Lösungsversuch, mit einem Beitrag zur Apokalypse von J. Frey (WUNT 67; Tübingen, 1993) 204-9.

${ }^{7}$ Diese Bemerkung wird durch die folgende textkritische Beobachtung unterstüzt, welche die frühe Verbreitung des Joh in Ägypten beweist. Aller Wahrscheinlichkeit nach stammen die Papyri 52, 66 und 90, die alle Auszüge aus der joh Passionsgeschichte enthalten, aus dem 2. Jh. 'Diese dreifache Bezeugung eines neutestamentlichen Textes aus dem 2. Jahrhundert ist einzigartig', schreibt zu Recht K. Aland, 'Der Text des Johannes-Evangeliums im 2. Jahrhundert', in: Studien zum Text und zur Ethik des Neuen Testaments (FS H. Greeven; hg. von W. Schrage; BZNW 47; Berlin/New York, 1986) 1. 
zugeordneten Überschriften erfüllen eine wichtige hermeneutische Funktion. Am Beispiel des Evangeliums sollen drei wichtige Funktionen aufgezeigt werden, die die Überschrift ausübt.

Die Überschrift hat zunächst eine benennende Funktion. Sie bildet 'ein Unterscheidungszeichen, das ein Werk in seiner Individualität zu identifizieren ermöglicht'. ${ }^{8}$ Das Evangelium nach Johannes und die drei Briefe des Johannes wurden mit Überschriften versehen, damit sie entweder bei ihrer öffentlichen Vorlesung im Gottesdienst oder in den Gemeindearchiven identifiziert werden konnten. ${ }^{9}$ Dass solches überhaupt notwendig wurde, weist daraufhin, dass die johanneischen Schriften zu öffentlichen Dokumenten wurden und dass sie in vielen bedeutenden christlichen Gemeinden des zweiten Jahrhunderts schnell bekannt wurden.

Die Überschrift hat des weiteren eine hinweisende Funktion. Sie gibt einen Hinweis auf den Inhalt des Werkes. Die Tatsache, dass

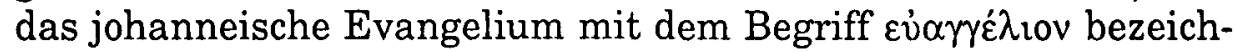
net wurde, ist eine Entscheidung von grosser hermeneutischer Tragweite. Dieser Ausdruck erscheint in den johanneischen Schriften selbst niemals. Durch diese Benennung des vierten Evangeliums greift der Herausgeber auf einen Begriff zurück, der zwar der johanneischen Tradition unbekannt ist, der aber bereits eine lange Geschichte hatte und zahlreiche Assoziationen im Gedächtnis der ersten Christen auslöste. Dieser Ausdruck, der seit Beginn des zweiten Jahrhunderts verwendet wurde, um die Erzählungen vom Leben Jesu zu benennen, ${ }^{10}$ ist zum ersten Mal wahrscheinlich zur Bezeichnung von Mk und, in seinem Gefolge, von Mt und Lk gebraucht worden. Durch diese Überschrift wurde das Johannesevangelium diesem Typ Literatur zugeordnet und damit faktisch in Beziehung zu den bereits bestehenden Erzählungen gesetzt.11

Der präpositionale Ausdruck $\kappa \alpha \tau \dot{\alpha}$ 'I $\omega \alpha \dot{v} v \eta v$ erfüllt eine dritte Funktion der Überschrift. Er löst ein Rätsel des Textes, nämlich dasjenige der Identität seines mutmasslichen Autors. ${ }^{12} \mathrm{~K} \alpha \tau \dot{\alpha}$ 'I $\omega \alpha \dot{v}$ v v kann nicht einfach durch einen Genitiv ersetzt werden. Die

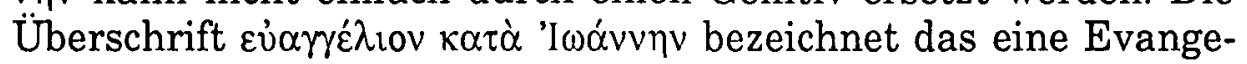

${ }^{8}$ Hallyn/Jacques, 'Aspects du paratexte', 204 (s. Anm. 5).

$9 \mathrm{Vgl}$. Hengel, Evangelienüberschriften, 33-40 (s. Anm. 6).

${ }^{10} \mathrm{Vgl}$. Hengel, Evangelienüberschriften, 8-28 (s. Anm. 6). G. Strecker, Literaturgeschichte des Neuen Testaments (UTB 1682; Göttingen, 1992) 123-8 bietet eine Bibliographie dazu und den Stand der Diskussion an.

11 Vgl. Hengel, Frage, 208-9 (s. Anm. 6).

12 Diese dritte Funktion der Überschrift wirft die sogenannte 'johanneische Frage' auf. Allerdings ist es im Rahmen dieses Aufsatzes, der literaturwissenschaftlich orientiert ist, weder möglich noch angebracht, diesen historischen Streitpunkt zu diskutieren. 
lium, aber so, wie es von der Person namens Johannes formuliert wurde. Indem der Name Johannes auf die drei johanneischen Briefe ausgeweitet wurde, schuf die verlegerische Instanz ein Korpus, das sich im christlichen Kanon nach und nach durchsetzen sollte.

Die Überschrift erfüllt also eine wichtige hermeneutische Funktion. Sie ermöglicht die Identifizierung und Zitierung des Werkes. Sie weist ihm einen Platz innerhalb der bereits bestehenden frühchristlichen Literatur zu. Sie bindet es an eine berühmte Person, die seine Autorität garantiert. Sie weist darauf hin, wie das Werk gelesen werden soll. Kurz, analog zu Joh 21 sichert die Überschrift die Verkirchlichung zunächst des Evangeliums und anschliessend der Briefe.

\section{DIE RELECTURE EINES LITERARISCHEN WERKES DURCH EIN ANDERES LITERARISCHES WERK}

Der zweite Typ von Relecture, auf den wir hinweisen möchten, besteht in der Relecture eines Werkes durch ein anderes. Dieser Vorgang entsteht dann, wenn die Rezeption eines Werkes solche Probleme aufwirft, dass sie zur Entstehung eines neuen Werkes führt, das klärt, wie das erste Werk richtig verstanden werden soll. Dieser Fall ist bereits im Alten Testament belegt: Das Sirachbuch, das hebräische Buch Daniel (Dan 1-12) oder die Pescharim von Qumran versuchen, den Sinn der prophetischen Verkündigung in einer veränderten geschichtlichen Situation nochmals neu zu formulieren.13 Der Vorgang findet sich ebenso in der paulinischen Tradition; denken wir nur an das Verhältnis zwischen dem ersten und zweiten Brief an die Thessalonicher. ${ }^{14}$ Unserer Meinung nach gehört die Frage nach dem Verhältnis zwischen dem vierten Evangelium und dem ersten Johannesbrief zu diesem Typ von Relecture. Die folgenden Überlegungen sollen diese Hypothese stützen.

Der Autor von 1 Joh verfolgt sein Ziel, indem er zwei Kategorien des Wissens einander gegenüberstellt: einerseits das traditionelle kirchliche Wissen, formuliert in der Form von kurzen Thesen, die

13 Steck, 'Prophetenauslegung', 199-205 (s. Anm. 4).

14 Zum Verhältnis zwischen dem ersten und dem zweiten Thessalonicherbrief vgl. W. Trilling, Der zweite Brief an die Thessalonicher (EKK 14; Zürich u.a., 1980) 21-32; ders., Untersuchungen zum 2. Thessalonicherbrief (EThSt 27; Leipzig, 1972). Stand der Diskussion bei U. Schnelle, Einleitung in das Neue Testament (UTB 1830; Göttingen, 1994) 365-77. Ein ähnliches Phänomen der Relecture stellt das Verhältnis zwischen dem Kol und dem Eph dar. 
durch die berühmten Formeln 'wir wissen', 'wir haben gehört' eingeleitet werden, und andererseits das heterodoxe Wissen, signalisiert durch die Ausdrücke 'wer sagt' oder 'wenn wir sagen'. ${ }^{15}$ Diese Art einer sehr typisierten Argumentation zeigt, dass der Inhalt des traditionellen Wissens in einer Situation des Konflikts reformuliert werden muss. Der erste Johannesbrief ist demnach als Leseanleitung konzipiert, die das richtige Verstehen der überlieferten Tradition ermöglichen soll. Der Brief liest die ihm vorausgehende Tradition noch einmal.

Sollte 1 Joh tatsächlich als ein Akt der Relecture der johanneischen Tradition verstanden werden, können wir nicht noch einen Schritt weitergehen und behaupten, dass es sich um eine Relecture des vierten Evangeliums handelt? Unserer Meinung nach lässt sich eine erste Antwort auf diese Frage formulieren, indem man die äusserst umstrittene Frage nach der literarischen Gattung von 1 Joh zu beantworten sucht.16 In der Geschichte seiner Rezeption wurde 1 Joh durchgehend als Brief verstanden. ${ }^{17}$ Die Forschung hat allerdings mit Recht auf das Fehlen eines konstitutiven Elements der Gattung des Briefes bei 1 Joh hingewiesen, nämlich des Briefformulars. Diese unbestreitbare Beobachtung bleibt allerdings solange von geringer Tragweite, als nicht diejenigen Elemente von 1 Joh sorgfältig geprüft werden, die an die Stelle des klassischen epistolaren Formulars treten. Aufschlussreich sind folgende Beobachtungen: Der Beginn des Briefes umfasst einen Prolog (1 Joh 1.1-4), der an denjenigen des Evangeliums erinnert; der Schluss von 5.13 nimmt denjenigen des Evangeliums auf (Joh 20.31); auf diesen Abschluss folgt seinerseits ein vermutlich sekundärer Epilog (5.14-21; vgl. Joh 21)! Unter der plausiblen Voraussetzung, dass 1 Joh nach dem vierten Evangelium anzusetzen ist, 18 besagt dieser Sachverhalt, dass 1 Joh die literarische Struktur des Evangeliums bewusst imitiert. Durch

15 Vgl. H.-J. Klauck, Die Johannesbriefe (EdF 276; Darmstadt, 1991) 55-6, im Gefolge von O. A. Piper, 'John and the Didache of the Primitive Church', JBL 66 (1947) 437-51; F. Vouga, Die Johannesbriefe (HNT 15/3; Tübingen, 1990) 9-11.

16 Bestandesaufnahme der Diskussion bei Klauck, Johannesbriefe, 68-74 (s. Anm. 15). Siehe insb. R. E. Brown, The Epistles of John (AncB 30; New York, 1982) 86-92.

$17 \mathrm{Vgl}$. etwa den Titel, der $1 \mathrm{Joh}$ seit dem 2. Jh. gegeben wurde; vgl. auch den Kanon Muratori (vgl. Klauck, Johannesbriefe, 22ff. [s. Anm. 15]) oder auch die Notiz zu Papias und 1 Joh bei Euseb Hist.Eccl. 3.39.17.

${ }^{18}$ Ein gewisser Konsens herrscht über die Reihenfolge Joh-1 Joh. Die einzigen erwähnenswerten Ausnahmen: G. Strecker, 'Die Anfänge der johanneischen Schule', NTS 32 (1986) 3147; U. Schnelle, Antidoketische Christologie im Johannesevangelium. Eine Untersuchung zur Stellung des vierten Evangeliums in der johanneischen Schule (FRLANT 144; Göttingen, 1987) 65-83; ders., Einleitung, 500, 503-4, 519-22 (s. Anm. 14). Eine ausführliche Diskussion der Problematik findet sich bei Brown, Epistles, 30-5 (s. Anm. 16). 
seine Architektur will der Text den Eindruck erwecken, dass er in einem besonderen Verhältnis zum Evangelium steht und in $\mathrm{Be}-$ ziehung zum Evangelium gelesen werden will.19

Die Frage nach dem Verhältnis zwischen 1 Joh und dem vierten Evangelium soll weiter vertieft werden. Fragen wir im Rahmen unserer eingangs formulierten Hypothese: Wie lässt sich der hier zutage tretende Prozess der Relecture charakterisieren? Eine Antwort auf diese Frage ist dann möglich, wenn wir einen mittlerweile klassischen Sachverhalt hinsichtlich 1 Joh in den Blick nehmen und nach seiner Bedeutung fragen. Die Forschung hat öfters auf das Phänomen hingewiesen, dass sich das Verhältnis zwischen 1 Joh und dem vierten Evangelium durch grosse Ähnlichkeiten und zugleich durch nicht weniger bedeutende Unterschiede auszeichnet; dies betrifft den Stil, die verwendete Begrifflichkeit und die behandelten Themen. ${ }^{20}$ M.E. darf dieser zweifache Befund von Übereinstimmung und Differenz nicht als eine Alternative verstanden werden, die zugunsten der einen oder anderen Seite aufzuheben ist.21 Diese Spannung zwischen Ähnlichkeit und Unterschiedlichkeit beschreibt vielmehr exakt die Situation der Relecture. Das bedeutet, dass eine bestehende Äusserung des Glaubens aufgenommen wird - hierin besteht die Ähnlichkeit -, um danach aufgrund von Aporien, in die diese Äusserung geführt hatte, aber auch aufgrund der neuen Situation, die diese Äusserung ausgelöst hatte, neu formuliert zu werden - hier manifestiert sich die Differenz. Die Relecture ist nicht als ein einfacher Vorgang der Wiederholung zu verstehen; sie führt vielmehr eine Rekontextualisierung durch.22 Ich möchte im folgenden an zwei bekannte Beispiele dieses Vorgangs erinnern.

19 An diesem Punkt der Argumentation verdient eine Beobachtung Aufmerksamkeit. Die Tatsache selbst, dass in der johanneischen Schule eine neue literarische Gattung entsteht, besagt, dass das Evangelium - trotz der verschiedenen Redaktionen, denen es unterzogen wurde - nicht mehr für Verbesserungen und Ergänzungen empfänglich war. Es stellt von nun an einen geschlossenen Text dar, es nimmt den Rang einer Schrift ein.

20 Diskussion bei Klauck, Johannesbriefe, 88-99 (s. Anm. 15). Vgl. insbesondere H. Conzelmann, '"Was von Anfang war"', in: ders., Theologie als Schriftauslegung (BEvTh 65; München, 1974) 207-14; G. Klein, " Das wahre Licht scheint schon." Beobachtungen zur Zeit- und Geschichtserfahrung einer urchristlichen Schule', ZThK 68 (1971) 261-326.

21 Die These, wonach das Evangelium und die Briefe das Werk eines einzigen Autors seien (so Hengel, Frage, 306-25 [s. Anm. 6]), verkennt die dialektische Beziehung zwischen Joh und den joh Briefen. Das ist aber auch der Fall, wenn behauptet wird, die joh Briefe verräten die theologische Auffassung des Joh (Stand der Diskussion bei E. Lohse, Grundriss der neutestamentlichen Theologie [ThW 5; Stuttgart u.a., 1974] 142-4). Zum ganzen Problem siehe Brown, Epistles, 14-30 (s. Anm. 16).

22 Mit dem Begriff der Rekontextualisierung wird der französische Begriff der recadrage wiedergegeben. 
Das erste Beispiel ist selbstverständlich der Prolog von $1 \mathrm{Joh}$. Wie neulich Brown und anschliessend Klauck gezeigt haben,23 gewinnt dieser Eröffnungstext sofort ein spezifisches Profil, wenn er zum einen auf dem Hintergrund des Prologs des vierten Evangeliums gelesen wird und wenn zum anderen festgestellt wird, dass er in ausserordentlich knapper Weise Schlüsselbegriffe des Evangelienprologs aufnimmt und sie neu kontextualisiert. So wird der Ausdruck Év $\alpha \rho \chi \eta ़$ von Joh 1.1 durch die Wendung $\dot{\alpha} \pi$ ' $\dot{\alpha} \rho \chi \hat{\eta} \varsigma$ von 1 Joh 1.1 neu bestimmt; und der Begriff $\lambda$ ó ${ }^{\circ} \varsigma$ des johanneischen

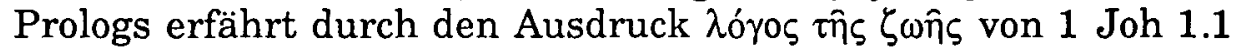
eine neue Wertung. Das zweite Beispiel ist durch das Liebesgebot gegeben. Dieses in Joh 13.34 als neu qualifizierte Gebot wird in 1 Joh 2.7-8 als zugleich alt, d.h. seit langem bekannt, und dennoch neu - hierin der vom Evangelium vorgenommenen theologischen Qualifizierung entsprechend - dargestellt. ${ }^{24}$ Zahlreiche weitere Beispiele für diesen Prozess der Rekontextualisierung könnten hier aufgeführt und diskutiert werden, so etwa die Figur des Parakleten, die Symbolik von Licht und Finsternis, die Bedeutung des Todes Christi, die Parusie, etc.

Ziehen wir ein Fazit. Wie 1 Joh vermuten lässt, hat der johanneische Kreis einen Interpretationskonflikt durchgemacht, der sich auf zentrale Aussagen des Evangeliums konzentrierte. Dieser Konflikt resultierte sowohl aus der dem vierten Evangelium inhärenten Ambivalenz als auch aus neuen geschichtlichen Situationen, denen die johanneische Bewegung ausgesetzt war. Um zu zeigen, wie die johanneische Tradition zu verstehen sei, verfasste die johanneische Schule einen Brief, der strittige Fragen beantworten und eine Leseanweisung zur Entschlüsselung des Evangeliums darstellen sollte. Dieser Relecture-Vorschlag ist der Versuch, die allen bekannten und von allen akzeptierten Aussagen, d.h. das Evangelium, neu zu strukturieren, indem er diese auf den Anfang der joh Tradition zurückbezieht. Der von der johanneischen Tradition postulierte Anfang25 wird zum regulativen Prinzip ihrer Interpretation und ihrer Entwicklung.

Wir wollen uns nun dem vierten Evangelium selbst zuwenden und klären, inwiefern es von internen Relecture-Prozessen geprägt ist.

$23 \mathrm{Vgl}$. Klauck, Johannesbriefe, 94-7 (s. Anm. 15); ders., Der erste Johannesbrief (EKK 23/1; Zürich u.a., 1991) 54-78; Brown, Epistles, 151-87 (s. Anm. 16). 16).

24 Vgl. Klauck, Der erste Johannesbrief, 120-4 (s. Anm. 23); Brown, Epistles, 264-7 (s. Anm.

25 Zum Ausdruck $\dot{\alpha} \pi$ ' $\dot{\alpha} \rho \chi \hat{\eta} \varsigma$ vgl. Conzelmann, 'Anfang' (s. Anm. 20); Brown, Epistles, 97-100 (s. Anm. 16). 


\section{DER PARATEXT: PROLOG UND EPILOG}

Die Theorie des Paratextes - die obige Diskussion der Evangelienüberschriften war eine erste Illustration dazu - erweist sich als sehr fruchtbar für denjenigen, der sich für das Phänomen der Relecture interessiert. Was ist unter einem Paratext zu verstehen? 'Der Paratext umfasst eine Summe von verschiedenen Zeichen, die einen bestehenden Text vorstellen, einrahmen, einführen, unterbrechen oder abschliessen [. . .]. Der Paratext übt im allgemeinen eine Funktion der Begleitung oder der Einrahmung eines anderen Textes aus.'26 Als Begleittext drückt der Paratext immer eine Stellungnahme zu einem ersten Text aus; er artikuliert eine Weise der Relecture. Der das Evangelium eröffnende Prolog und der es abschliessende Epilog gehören zu diesem Texttyp. Folgende Frage muss hier gestellt werden: In welchem Sinnverhältnis stehen der Prolog und der Epilog zur johanneischen Erzählung? Welche Funktion nehmen sie gegenüber dem Korpus des Evangeliums ein?

Beginnen wir mit dem Prolog. ${ }^{27}$ Unter einem literaturwissenschaftlichen Gesichtspunkt ist der Prolog eine Textsorte, die 'sowohl einen Rückblick der Lektüre auf die Schrift als auch eine Antizipation der Schrift auf die Lektüre beinhaltet. Eine Vorrede kann darauf zielen, dem Werk einen Sinn zuzusprechen [. . .]. Die Vorrede ist ein Kontrollinstrument des Entschlüsselns. Sie steuert die Lektüre, verteidigt den Text gegen Unverständnis und falsche Interpretationen.'28 Diese Definition der Gattung des Prologs ist nicht neu. Sie ist bereits in der antiken griechischen und lateinischen Literatur belegt, insbesondere bei Aristoteles, ${ }^{29}$ nach dem das Vorwort die Zuhörerschaft über die Absichten eines Werkes aufklären und ihr dadurch die Mittel zur Verfügung stellen muss, um dessen Gegenstand und Verlauf zu verstehen.

Stimmt man dieser Definition zu, dann muss der johanneische

${ }^{26}$ Hallyn/Jacques, 'Aspects du paratexte', 202 (s. Anm. 5).

27 Zum Verhältnis zwischen dem Prolog und dem Korpus des Evangeliums siehe meinen Artikel 'Le prologue, seuil du quatrième évangile', $R S R 83$ (1995) 21 7-39.

28 Hallyn/Jacques, 'Aspects du paratexte', 210-11 (s. Anm. 5). Die vollständige Passage auf französisch lautet folgendermassen: 'Un discours préfaciel peut viser à imposer un sens à l'œuvre, à y faire voir le résultat d'une production orientée, fût-ce obscurément, vers l'énonciation de ce sens. Plus modestement, sa fonction peut consister à repragmatiser le discours écrit, ce message en lui-même essentiellement dépragmatisé. Dans les deux cas, le prédiscours est un instrument de contrôle du décodage. Il dirige la lecture, défend le texte contre l'incom. préhension et les interprétations erronées. Protégeant un orphelin, il supplée à l'absence du père de l'écrit, évoquée déjà par Platon.'

29 Vgl. D. E. Smith, 'Narrative Beginnings in Ancient Literature and Theory', Semeia 52 (1990) 33, 41; Aristoteles Rhetorik 3.14.12-19, 22-5. 
Prolog als ein Eintreten in die Lektüre verstanden werden, welches die richtige Entschlüsselung der nachfolgenden Erzählung ermöglicht. Dieses Eintreten in die Lektüre ist nicht auf der gleichen Ebene wie die Erzählung selbst zu situieren, sondern setzt bereits eine Reflexion über diese Erzählung voraus. Dieser metareflexive Charakter des Prologs ${ }^{30}$ macht ihn zu einem klassischen Beispiel eines Relecture-Phänomens.

Diese Differenz der Ebene zwischen dem Prolog und dem Korpus des Evangeliums - eine Differenz, die den metareflexiven Charakter des Prologs unterstreicht - kann sowohl auf literarischer wie auf theologischer Ebene verifiziert werden. Nehmen wir zunächst die literarische Ebene in den Blick. Während von Joh 1.19 an das Evangelium den Weg der geschichtlichen Erzählung einschlägt, indem es die Figur des Täufers und seines Zeugnisses zur Sprache bringt, liest sich der Prolog als ein Hymnus, der in der Sprache des Mythos das Kommen des präexistenten Logos in die Welt preist. ${ }^{31}$ Eine Verschiebung zeigt sich auch auf der theologischen Ebene. Die im Prolog behandelten grossen Themen - der Übergang von der Präexistenz zur Inkarnation, die vermittelnde Aktivität des Logos bei der Schöpfung, die radikale Formulierung der Inkarnation - werden nicht direkt im Erzählkorpus wiederaufgenommen. Dasselbe gilt für wichtige Schlüsselbegriffe, von denen in erster Linie der christologische Titel des Logos zu erwähnen ist. ${ }^{32}$ Allerdings fehlen intentionale Bezüge zur nachfolgenden Erzählung nicht einfach, obschon der Prolog als ein spezifischer Teiltext zu verstehen ist: Joh 1.1 und 20.28, die beide auf ihre Weise die Göttlichkeit des Sohnes aussagen, bilden eine Inklusion. Das Zeugnis des Täufers, das im Prolog in zwei Durchgängen formuliert ist, eröffnet die Erzählung. Schliesslich lässt sich leicht zeigen, dass eine Anzahl von Begriffen, die im Prolog zum ersten Mal erwähnt sind, in der nachfolgenden Erzählung aufgenommen und entwickelt werden.

Diese Bemerkungen machen deutlich, dass der Prolog weder die erste Episode des Evangeliums noch die Zusammenfassung seiner dramatischen Handlung ${ }^{33}$ noch sein theologisches Summar

30 Der metareflexive Charakter des Prologs wird nachdrücklich hervorgehoben von $M$. Theobald, Die Fleischwerdung des Logos. Studien zum Verhältnis des Johannesprologs zum Corpus des Evangeliums und zu 1 Joh (NTA.NF 20; Münster, 1988) insb. 296-399, 438-93.

31 Zum mythologischen Charakter des Prologs und dessen Interpretation vgl. zuletzt $\mathrm{H}$. Weder, 'Der Mythos vom Logos (Johannes 1)', in: ders., Einbliche ins Evangelium. Exegetische Beiträge zur neutestamentlichen Hermeneutik (Göttingen, 1992) 401-34.

32 Ein Inventar findet sich in meinem Artikel 'Prologue', 220-2 (s. Anm. 27).

33 Der Begriff der dramatischen Handlung entspricht dem französischen Begriff 'intrigue' und dem englischen 'plot'. 
darstellt. Der Prolog erfüllt vielmehr die Funktion, den hermeneutischen Rahmen festzulegen, innerhalb dessen die Erzählung gelesen werden soll. Noch bevor sich die Erzählung zu entwickeln beginnt und dank des Sinnüberschusses, der für einen Mythos charakteristisch ist, erhellt der Prolog das Hauptanliegen und die Bedeutung der Geschichte, die im nachfolgenden erzählt werden wird. Um es ein wenig riskant $z u$ formulieren: Indem der Logos zum absoluten und begründenden Anfang in Beziehung gesetzt und darauf die Bewegung von der Präexistenz zur Inkarnation nachgezeichnet wird, will der Prolog zu bedenken geben, dass der Mensch Jesus, der im Zentrum der nachfolgenden Erzählung stehen wird, die Verdoppelung Gottes ${ }^{34}$ inmitten der Welt darstellt.

Wenden wir uns nun Joh $21 \mathrm{zu}$, dem Epilog des Evangeliums. ${ }^{35}$ Gemäss der Theorie des Paratextes 'kommt dem Epilog bzw. Nachspiel oder Nachwort üblicherweise die Funktion zu, eine (stabile) Situation kurz zu schildern, die zeitlich nach dem eigentlichen Schluss liegt und aus ihm folgt'. ${ }^{36}$ Diese Definition lässt sich in unserem Fall verifizieren. Nach der im wesentlichen einhelligen Auffassung der Forschung ist Joh 21 eine spätere Hinzufügung an das Korpus des Evangeliums. ${ }^{37}$ Das Kapitel beansprucht diesen Status insofern explizit, als es den Schluss des vorausgehenden Textes respektiert, nämlich die abschliessende Wendung Joh 20.30-1. Es ist also ausserhalb der eigentlichen Erzählung angesiedelt, steht aber dennoch in einer engen Beziehung zur Erzählung - und dies auf verschiedene Weise: Das Kapitel imitiert den. johanneischen Text, indem es das Schema 'Zeichen - Rede' übernimmt und einen zweiten Schluss gestaltet. Das Kapitel benutzt im weiteren das Vorgehen des Verweisens: Es positioniert sich explizit in der Folge von Joh 20 (Joh 21.1: $\mu \varepsilon \tau \grave{\alpha} \tau \alpha \hat{v} \tau \alpha$. . . $\pi \alpha ́ \lambda_{\imath v}$ ); es numeriert die Erscheinungen (Joh 21.14); es benennt den Lieblingsjünger mit den in Joh 13.23 verwendeten Termini; es

34 Der Begriff der Verdoppelung Gottes (dédoublement de Dieu) wurde zuerst von P. Beauchamp vorgeschlagen; vgl. dazu X. Léon-Dufour, Lecture de l'évangile selon Jean (chapitre 14) (Parole de Dieu; Paris, 1988) 1.59.

${ }^{35} \mathrm{Zu}$ Joh 21 siehe meinen Artikel 'La rédaction finale de l'évangile selon Jean (à l'exemple du chapitre 21)', in: ders., Miettes exégétiques (MoBi 25; Genève, 1991) 253-79.

36 G. Genette, Palimpseste. Die Literatur auf zweiter Stufe (Aesthetica; edition suhrkamp. NF 683; Frankfurt am Main, 1993) 280 (das französische Original: Palimpsestes, 231 [s. Anm. 3]).

37 Stand der Forschung und wichtigste Literatur bei J. Becker, Das Evangelium nach Johannes. Kapitel 11-21 (ÖTBK 4/2; 3. Auf1.; Gütersloh/Würzburg, 1991) 758-60; R. Schnackenburg, Das Johannesevangelium. Kommentar zu Joh 13-21 (HThK 4/3; Freiburg u.a., 1975) 406-17; Schnelle, Einleitung, 555-6 (s. Anm. 14). 
stellt der dreifachen Verleugnung des Petrus seine dreifache Rehabilitierung entgegen, u.s.w.

Worin besteht also der Sinn dieses Epilogs, der sich in Beziehung zur vorausgehenden Erzählung setzt, um sich zugleich explizit von ihr zu unterscheiden? Die in Joh 21 dargelegte Situation resultiert aus der abgeschlossenen Erzählung und bezieht Stellung zu dieser Erzählung, indem sie die aus der Erzählung resultierenden Folgerungen in einer neuen geschichtlichen Situation noch einmal neu bewertet. Worin besteht diese Neubewertung? Inwiefern liegt eine Relecture vor? Während die zentrale Thematik des Korpus des Evangeliums die Christologie darstellt, ist die Thematik von Joh 21 deutlich ekklesiologisch. Der Christus offenbart nicht mehr sich selbst, sondern klärt die Bedeutung von zwei Schlüsselfiguren, des Petrus und des Lieblingsjüngers, für die nachösterliche Zeit. Genauer: das Verhältnis zwischen Petrus und dem Lieblingsjünger wird zugunsten des ersteren neu bestimmt. Sowohl der Apostelfürst, dessen Portrait im Korpus des Evangeliums nicht gerade vorteilhaft ausgefallen war, als auch der Lieblingsjünger werden in ihrer zukünftigen Funktion für die gesamte Kirche positiv dargestellt. Petrus wird in seine Rolle als universaler Hirte und ruhmvoller Märtyrer eingeführt, während der Lieblingsjünger zum Autor eines Zeugnisses, des vierten Evangeliums, wird, das zur Auferbauung aller Glaubenden dienen soll. In diesem Sinne führt die im Kap. 21 unternommene Relecture eine Repragmatisierung des Evangeliums durch. Indem die Relecture durch das Wort des johanneischen Christus die Funktion des Petrus anerkennt, bindet sie die Funktion des Lieblingsjüngers an diejenige des Petrus. Das Evangelium setzte den Christus in die Erzählung um, während der Epilog die Verkirchlichung der Erzählung garantiert.

\section{DER PROZESS DER INTERNEN RELECTURE}

$\mathrm{Zu}$ Beginn unserer Ausführungen haben wir den Begriff der Relecture folgendermassen definiert: Ein Relecture-Prozess liegt dann vor, wenn ein erster Text die Bildung eines zweiten Textes hervorruft und wenn dieser Text seine volle Verständlichkeit erst im Bezug zum ersten Text gewinnt. Bisher haben wir uns dem Phänomen der Relecture angenähert, indem wir einerseits den Paratext des Evangeliums, das heisst die Überschrift, den Prolog und den Epilog, und anderseits den ersten Johannesbrief, der als Leseanleitung zum Evangelium zu verstehen ist, in den Blick genommen haben. Nun soll das Korpus des Evangeliums selbst 
untersucht und beobachtet werden, ob darin vergleichbare Phänomene ausfindig gemacht werden können.

Unsere These lautet, dass das Phänomen der Relecture in verschiedenen Formen auch im Evangelium selbst wahrnehmbar ist. Die Tragweite dieser These, wenn sie sich verifizieren lässt, ist beträchtlich, denn sie erlaubt es, die johanneische Literarkritik in einer anderen Perspektive wahrzunehmen. Um allerdings Verwirrungen und Missverständnissen vorzubeugen, seien vorausgehend die folgenden zwei Punkte kurz festgehalten: Zum einen muss das Phänomen der Relecture diszipliniert gehandhabt werden; es bezeichnet weder die Gesamtheit der Arbeitsweisen der johanneischen Schule noch a fortiori die Gesamtheit der redaktionellen Arbeit. ${ }^{38}$ Die Relecture ist und bleibt eine johanneische Arbeitsweise neben weiteren. Zum anderen lässt sich das Phänomen der Relecture nicht auf eine einzige Etappe in der Geschichte der Konstitution des Textes eingrenzen, sondern ist - um die vertrauten Begriffe der joh Exegese zu verwenden - sowohl für die Ebene des Evangelisten als auch für diejenige der Schlussredaktion bezeugt. 39 Ohne Anspruch auf Vollständigkeit möchten wir auf drei unterschiedliche Arten der Relecture hinweisen.

Zum ersten - dieses Phänomen stellt allerdings einen ganz normalen literarischen Vorgang dar - sind die in die Erzählung eingefügten Glossen zu erwähnen. ${ }^{40}$ Die einen erhellen ein Detailproblem der Erzählung; so etwa die Notiz zur vermeintlichen Tauftätigkeit Jesu in Joh 4.2. Andere kommentieren die Erzählung theologisch (so Joh 4.44; 7.39b; 12.16), wieder andere rekontextualisieren das theologische Programm der johanneischen Schrift (so die eschatologischen Notizen in Joh 5.28-9; 6.39, 40, 44, 54; 12.48). Diese Glossen dürfen von der Exegese nicht theologisch disqualifiziert oder ignoriert werden. Sie sind auf ihre Weise ein Reflex der kontinuierlichen Arbeit der johanneischen Schule.

Zum zweiten ist die interpretative Entfaltung von Erzählungen zu erwähnen. Es ist hinreichend bekannt, dass die Tempelreinigung nicht nur eine einzige Auslegung enthält (Joh 2.14-17), sondern dass diese erste Lektüre durch eine zweite verdoppelt

\footnotetext{
${ }^{38} \mathrm{Zu}$ der johanneischen Schule und ihrer interpretatorischen Arbeit vgl. J. Becker, Das Evangelium nach Johannes. Kapitel 1-10 (ÖTBK 4/1; 3. Aufl.; Gütersloh/Würzburg, 1991) 4750 (Stand der Diskussion und Literatur). Andere Arbeitsweisen der johanneischen Schule werden z.B. in den folgenden Artikeln dargestellt: J. Becker, 'Joh 3,1-21 als Reflex johanneischer Schuldiskussion', in: Das Wort und die Wörter (FS G. Friedrich; hg. von H. Balz/ S. Schuiz; Stuttgart, 1973) 85-95; Schnelle, 'Johanneische Schule' (s. Anm. 2).

39 Das Phänomen lässt sich allerdings auch auf der vorredaktionellen Ebene beobachten.

40 Vgl. R. Schnackenburg, Das Johannesevangelium. Einleitung und Kommentar zu Kap. 14 (HThK 4/1; 3. Aufl.; Freiburg u.a., 1972) 36, 44, 60.
} 
wird (Joh 2.18-22). ${ }^{41}$ Auf ähnliche Weise erschöpft sich die Fusswaschung nicht in einer ersten Entschlüsselung (Joh 13.6-11); vielmehr wird in V. 12-17 eine zweite Lektüre vorgeschlagen.42 $\mathrm{Zu}$ diesem Typ von Entfaltung gesellt sich ein weiterer Interpretationsprozess, der sich in den grossen Texteinheiten wie z.B. Joh 5 und 6 findet. Die ausserordentlich knapp erzählte Wundergeschichte in Joh 5.1-9a ruft eine eigentliche Kaskade von aufeinanderfolgenden Lektüren hervor: das Streitgespräch (Joh 5.9b-18), die Rede über die Autorität des Sohnes (Joh 5.19-30) und die Rede über das Zeugnis (Joh 5.31-47).43 Dasselbe Phänomen lässt sich in Joh 6 beobachten. ${ }^{44}$ Auf die Wundergeschichte der Brotvermehrung (Joh 6.1-15) folgt eine ganze Reihe von Lektüren: zunächst eine zweite Wundergeschichte (Joh 6.16-21), ${ }^{45}$ dann eine Rede über das Brot (Joh 6.22-51a), anschliessend eine eucharistische Relecture (Joh 6.51b-58) und endlich die Szene mit den Jüngern (Joh 6.60-71).

Schliesslich soll ein letzter Fall von Relecture erwähnt werden, derjenige, bei dem eine Rede des johanneischen Christus in einer späteren Rede aufgenommen wird, um sowohl expliziert als auch rekontextualisiert zu werden. Die Abschiedsreden bieten die klassische Illustration für dieses Vorgehen. Die erste Abschiedsrede (Joh 13.31-14.31) wird in der dritten Abschiedsrede (Joh 16.4b-33) aufgenommen, die ihre Relecture darstellt.

Wie lässt sich diese Arbeit der Relecture näher beschreiben? Ich möchte vier Aspekte dieses Vorgangs aufzeigen. Ich werde mein Vorhaben anhand der Abschiedsreden darstellen, die von Andreas Dettwiler in dieser Perspektive auf ausgezeichnete Weise analysiert worden sind. 46

41 Zur Entstehungsgeschichte der Tempelreinigung vgl. Becker, Evangelium nach Johannes, 1.144-50 (s. Anm. 38); R. Bultmann, Das Evangelium des Johannes (KEK 2; 10. Aufl.; Göttingen, 1964) 85-6; Schnackenburg, Johannesevangelium 1.359-60 (s. Anm. 40).

42 Vgl. die klassische Hypothese von Bultmann, Evangelium des Johannes, 355-65 (s. Anm. 41). Vgl. auch ihre Aufnahme und Weiterführung bei Becker, Evangelium nach Johannes, 2.497-512 (s. Anm. 37); Schnackenburg, Johannesevangelium, 3.7-15 (s. Anm. 37).

43 Es lässt sich allerdings fragen, ob Joh $5.31-47$ noch zum Prozess der Relecture im strengen Sinne gehört.

$44 \mathrm{Zu}$ der interpretatorischen Bewegung in Joh 6 vgl. H. Weder, 'Die Menschwerdung Gottes. Überlegungen zur Auslegungsproblematik des Johannesevangeliums am Beispiel von Joh 6', in: ders., Einblicke, 363-400 (s. Anm. 31).

45 Die zweite Wundergeschichte sollte als Bestandteil des narrativen Konzepts von Joh 6 verstanden werden, auch wenn es sich dabei nicht um eine Relecture der Brotvermehrung im strengen Sinn handelt.

46 Vgl. A. Dettwiler, Die Gegenwart des Erhöhten. Eine exegetische Studie zu den johanneischen Abschiedsreden (Joh 13,31-16,33) unter besonderer Berücksichtigung ihres Relecture-Charakters (FRLANT 169; Göttingen, 1995). Eine synthetische Darstellung des Phänomens der Relecture ist auf den S. 44-52 zu finden. 
Erstens: Auch wenn die Arbeit der Relecture in der Schaffung eines zweiten Textes mündet, der den ersten Text aufnimmt und ihn weiter entwickelt, so beeinträchtigt dieser Prozess die Legitimität des ersten Textes dennoch nicht. Der erste Text wird sowohl in seiner literarischen Integrität als auch in seinem theologischen Aussagegehalt anerkannt. Er wird weder verbessert noch mit Glossen versehen. Das unbestreitbare und auffällige Zeichen dieses Respektes gegenüber dem ersten Text ist in dem von uns gewählten Beispiel die Bewahrung des berühmten Verses Joh 14.31c ('Steht auf, lasst uns von hier weggehen'), ein Vers, der den Respekt gegenüber der Abgeschlossenheit der ersten Abschiedsrede bezeugt. Gegenüber einer in der johanneischen Forschung zu oft vertretenen Auffassung ist zu betonen, dass das Verhältnis des zweiten Textes zum ersten Text nicht ein Verhältnis der Negation oder der Korrektur, ${ }^{47}$ sondern der Explizierung und der Vertiefung ist. Die durch den ersten Text geschaffene theologische Perspektive wird nicht aufgegeben, sondern im Gegenteil vom zweiten Text vorausgesetzt. Der Prozess der Relecture charakterisiert sich demnach durch die Entfaltung einer interpretativen Bewegung.

Zweitens: Es gilt zu sehen, dass der zweite Text von Beginn an als ein zweiter Text betrachtet wird. Die johanneische Schule hat ihn von Anfang an als einen zweiten Text gestaltet und ihm denjenigen besonderen Ort zugewiesen, der ihm im Rahmen der johanneischen Erzählung zukommt. V. 5 von Joh 16 ('Aber jetzt gehe ich weg zu dem, der mich gesandt hat, und niemand von euch fragt mich: "Wohin gehst du?"') bezieht sich explizit auf die Thematik der ersten Abschiedsrede (vgl. Joh 13.36: 'Simon Petrus sagt zu ihm: "Herr, wohin gehst du?"'),48 und V. 7 desselben Kapitels fasst ihre zentrale These zusammen ("Es ist zu eurem Vorteil, dass ich fortgehe'). Auf ähnliche Weise ist die zweite Interpretation der Fusswaschung ohne die ihr vorausgehende Erzählung unverständlich.

Drittens: Wie bereits bei Joh 21 erwähnt, realisiert sich die Arbeit der Relecture in zweifacher Weise. Sie ist zunächst durch eine Arbeit der Explikation charakterisiert. Bestimmte Motive werden aufgenommen, um später entfaltet zu werden. Während beispielsweise bereits die erste Abschiedsrede die glaubende Existenz als eine im Übergang von der Trauer zur Freude begriffene darstellte (Joh 14.1, 27, 28), so widmet Joh 16 diesem Aspekt nun

$47 \mathrm{Zu}$ diesem Aspekt vgl. meine forschungsgeschichtliche Stellungnahme in 'Rédaction finale', 257-8 (s. Anm. 35).

$48 \mathrm{Vgl}$. Dettwiler, Gegenwart, 219-20, 278 (s. Anm. 46). 
eine spezifische Reflexion (Joh 16.16-24). Die Arbeit der Relecture charakterisiert sich sodann durch eine Rekontextualisierung der behandelten Thematik. Während die zentrale Thematik der ersten Abschiedsrede christologisch ist und die Problematik des Weggangs Christi zu lösen versucht (die enigmatische Frage ist: 'Wohin gehst du?'), so ist die zentrale Thematik von Joh 16 anthropologischer und ekklesiologischer Natur. ${ }^{49}$ Genauer: Auch wenn als gültig angenommen wird, dass der Weggang des Christus notwendig und ertragreich ist, so bleibt es dennoch dabei, dass sich die Gemeinde dadurch verlassen fühlt und einer feindlichen Welt ausgesetzt sieht. Es geht also in der dritten Abschiedsrede um die ekklesiologische Bewahrheitung der christologischen These.

Viertens kann danach gefragt werden, welche Faktoren diesen für das vierte Evangelium so charakteristischen Prozess der Relecture in Gang gesetzt haben. Unserer Meinung nach muss man sich vor jedem monokausalen Erklärungsversuch hüten. Zunächst gilt es, auf die historischen Faktoren hinzuweisen, wobei diese allerdings in dialektischer Weise bedacht werden müssen. Gegen Ende des ersten Jahrhunderts waren die Beziehungen zwischen den johanneischen Gemeinden und der sie umgebenden Welt insbesondere der pharisäischen Synagoge - extrem gespannt. Aber diese Spannungen konnten ebensosehr durch die feindliche Umwelt wie durch das von den johanneischen Gemeinden verteidigte Überzeugungssystem provoziert worden sein. Das Evangelium ist eine die Krise bedingende und zugleich eine von dieser Krise bedingte Grösse. Es löst die Krise ebensosehr aus wie es auf sie antwortet. Es muss aber auch mit der theologischen Leidenschaft der johanneischen Schule gerechnet werden.50 Der Glaube wird nicht ein für allemal gedacht und formuliert. Er ruft nach einer Reflexion, welche die Aporien, die Risiken und die Implikationen des Glaubens zu klären versucht.

Wenn unsere Hypothese zur Relecture einige Plausibilität zu beanspruchen vermag, bedeutet dies, dass die johanneische Schule, deren wichtigstes Werk das vierte Evangelium ist, ein ausgeprägtes Bewusstsein für die Notwendigkeit der theologischen Arbeit und seiner Aktualisierung gehabt hat. Hat sie sich auch über die theologische Legitimität ihrer eigenen interpretativen Arbeit Gedanken gemacht? Diesen letzten Punkt möchte ich im folgenden kurz diskutieren.

$50 \mathrm{Zu}$ diesem wichtigen Aspekt vgl. Weder, Menschwerdung, 369 (s. Anm. 44). 


\section{DIE THEOLOGISCHE BEGRÜNDUNG DER RELECTURE}

Die Praxis der Relecture weist auf die Notwendigkeit des Erinnerns und zugleich auf seine ständige Erneuerung hin.51 Dieses Bewusstein für die Notwendigkeit des Erinnerns und seiner jeweils neuen Durchführung ist in der johanneischen Schule nicht implizit geblieben, sondern ist selbst zum Gegenstand eines intensiven Nachdenkens geworden. Ich möchte dies zeigen, indem ich abschliessend zwei Aspekte dieser Reflexion thematisiere. Der erste Aspekt betrifft die johanneischen Prolepsen des Erinnerns, während der zweite den Akteur dieses Erinnerns, den Parakleten, im Blick hat.

Das vierte Evangelium enthält drei Prolepsen, die dem Problem der Erinnerung gewidmet sind. Es sind dies Joh 2.22; 12.16 und 20.9:

-2.22 : 'Als er nun von den Toten auferweckt worden war, erinnerten sich seine Jünger, dass er dies gesagt hatte, und sie glaubten der Schrift und dem Wort, das Jesus gesagt hatte.'

- 12.16: 'Dies verstanden seine Jünger zunächst nicht; aber als Jesus verherrlicht war, erinnerten sie sich, dass dies über ihn geschrieben stand und dass sie ihm dies getan hatten.'

- 20.9: 'Denn sie verstanden die Schrift noch nicht, dass er von den Toten auferstehen müsse.'

Diese drei Passagen machen zwei Anmerkungen nötig.

Zum einen binden diese drei Passagen den Ursprung des Erinnerns an das Ereignis von Ostern. ${ }^{22}$ Die begründende Vergangenheit bringt ihre Bedeutung also nicht aus sich selbst hervor. Sie ist vielmehr Gegenstand einer erhellenden Retrospektive, die sich in diesem österlichen Wechsel herauskristallisiert. Der Glaube der Jünger begründet sich mit anderen Worten selbst nach dem Urteil des Evangeliums in einem Akt der Relecture, d.h. in der österlichen Relecture des irdischen Geschicks Christi. Im übrigen ist die Beobachtung aufschlussreich, dass im Johannesevangelium das Verb 'erkennen', wenn es von den Jüngern ausgesagt wird, normalerweise im Futur steht (vgl. Joh 13.7; 14.20).

Zum anderen sind an den drei zitierten Stellen das Erinnern und die Schrift miteinander verbunden. Das österliche und sinnstiftende Erinnern ist also zugleich Interpret der Schrift wie auch

51 Vgl. meinen Artikel 'Mémoire et relecture pascale dans l'évangile selon Jean', in: ders., Miettes exégétiques (MoBi 25; Genève, 1991) 299-316.

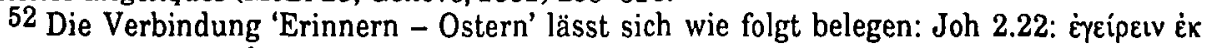

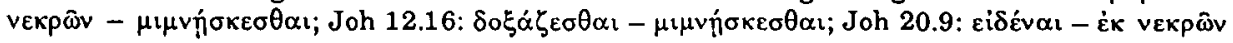
$\dot{\alpha} v \alpha \sigma \tau \hat{v} v \alpha$. 
von ihr her strukturiert. Nicht nur offenbart das Erinnern den Sinn des Geschicks Christi, sondern es lässt zugleich die Wahrheit der Schrift erscheinen und findet in dieser nun offenen Schrift die interpretativen Mittel vor. Im weiteren gilt es, auf eine Eigenheit des johanneischen Evangeliums aufmerksam zu sein. Die Schrift, auf die das Erinnern zurückgreift, ist gewiss die Bibel der ersten christlichen Gemeinden (Joh 2.17, 22; 12.16), aber sie umfasst ebenso die Worte Jesu (Joh $2.22 ; 14.26 ; 16.4) .{ }^{53}$ Das Erinnern bezeugt demnach die Worte Christi und interpretiert sie zugleich in österlicher Perspektive.

Die drei Prolepsen des Erinnerns ermöglichten es uns, zu entdecken, dass, unter einem johanneischen Gesichtspunkt gesehen, das richtige Verstehen der Person Christi untrennbar mit einem Akt der Relecture verbunden ist, dass das Erinnern nicht einfach blosse Restitution, sondern sinnproduzierende, erhellende Retrospektive ist. Welches ist demnach das handelnde Subjekt dieses Erinnerns?

Die johanneische These dürfte bekannt sein. Das handelnde Subjekt dieser erhellenden Retrospektive ist der Paraklet. Die fünf Worte über den Parakleten (Joh 14.16-17, 26; 15.26; 16.7-11, 13$15)^{54}$ zeigen, dass der Paraklet die gleichen Funktionen wie der Christus ausübt, dass er in gewisser Weise das nachösterliche Doppel des vorösterlichen Christus ist. Diese funktionale Identität darf aber nicht im Sinne einer Ranggleichheit verstanden werden. Was die Offenbarung betrifft, so bringt der Paraklet nichts Neues; er verdoppelt vielmehr das eschatologische Ereignis. Der Paraklet ist die im Vollzug begriffene Anamnese Jesu, die österliche Retrospektion des inkarnierten Christus. Oder um es mit den Worten dieses Aufsatzes zu formulieren: Der Paraklet ist das handelnde Subjekt der Relecture der Tradition Jesu. ${ }^{55}$

Einen letzten Punkt gilt es zu unterstreichen. Nehmen wir Joh 16 als Relecture von Joh 13.31-14.31 in den Blick, so ist es sehr aufschlussreich zu beobachten, wie Kap. 16 die Aussagen

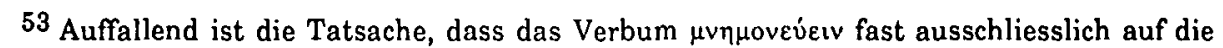
Worte des johanneischen Christus zurückverweist (Joh $15.20 ; 16.4)$. Dasselbe gilt für das johanneische hapaxlegomenon i்

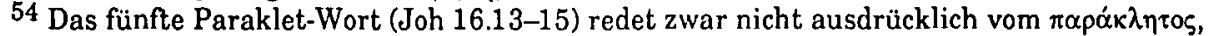
sondern von 'jenem' $\pi v \varepsilon \hat{v} \mu \alpha \tau \eta \hat{\zeta} \dot{\alpha} \lambda \eta \theta \varepsilon i \alpha \varsigma$. Dass aber dabei dieselbe Grösse wie in Joh 16.7-11 gemeint ist, dürfte unbestritten sein: Zum einen nimmt das Demonstrativpronomen غ̇keivos von V. 13 auf das vorausgehende غ̇кeivos von V. 8 Bezug, zum anderen machen Joh 14.16-17

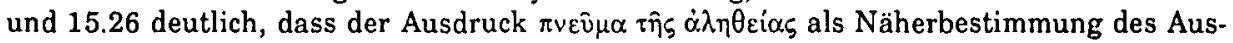

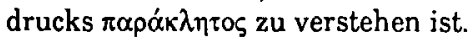

55 Es wäre hier angebracht, die Beziehung zwischen dem Parakleten und dem Lieblingsjünger zu klären, denn auch der Lieblingsjünger ist auf seine Weise ein handelndes Subjekt der Relecture der Tradition Jesu. 
zum Parakleten von Kap. 14 neu gelesen hat. Während die zwei Paraklet-Worte von Kap. 14 darauf insistieren, dass der Paraklet der gegenwärtige Christus nach Ostern (Joh 14.16) und das Subjekt der verstehenden Anamnese der Worte des irdischen Christus ist (Joh 14.26), erweitern die zwei Paraklet-Aussagen von Kap. 16 das Feld des Erinnerns. Das Kommen des Parakleten ist nicht mehr nur ein Ereignis des Erinnerns, sondern ein Ereignis, das ebenso die Gegenwart wie auch die Zukunft betrifft. Das vierte Wort über den Parakleten definiert seine Funktion im Verhältnis zum gegenwärtigen kó $\mu_{0}$ os. Der Paraklet ist der Urheber des eschatologischen Gerichts im Hier und Jetzt der nachösterlichen Zeit. Und das letzte Wort über den Geist der Wahrheit versteht das 'Hineinführen in die ganze Wahrheit' ausdrücklich nicht als ein Kennzeichen der vorösterlichen, sondern der nachösterlichen Zeit. Ja, die Eröffnung der ganzen Wahrheit betrifft nicht nur das richtige Begreifen der begründenden Vergangenheit, sondern schliesst nun die épxó $\mu \varepsilon v \alpha$ mit ein. Die Relecture besteht also nicht mehr einfach in einer erhellenden Retrospektive, sondern umfasst ebensosehr die Gegenwart wie die Zukunft. Die Notwendigkeit des Prozesses der Relecture findet demnach ihre letzte Rechtfertigung im Evangelium selbst. 